\title{
FROM MICROSCOPIC TO MACROSCOPIC DESCRIPTIONS OF COMPLEX SYSTEMS
}

\author{
M. Lachowicz \\ Institute of Applied Mathematics and Mechanics, \\ Faculty of Mathematics and Mechanics \\ University of Warsaw, Warsaw, Poland
}

\section{General statement}

The general approach that relates microscopic and nonlocal models with those at the macroscopic scale is considered. The macroscopic models are in terms of a system of reaction-diffusion equations. The micro-scale level is defined by a large number of interacting entities, and is in terms of a Markov jump process and related linear evolution equations. The intermediate scale (meso-scale) refers to the description of test-entities and is given in terms of nonlinear Boltzmann--type equations. Mathematical relationships between these three possible descriptions are proved and explicit error estimates are given.

\section{Details}

Usually the description of complex systems (e.g. biological populations) is carried out on a macroscopic level of interacting subpopulations within the system. Such an approach can be related to deterministic reaction-diffusion equations. They describe the (deterministic) evolution of densities of subpopulations of the system rather than the individual entities.

However, in many cases the descriptions on a micro-scale or a meso-scale of interacting entities (particles, cells, individuals, ...) seem to be more appropriate. The important feature of the microscopic level can be a nonlocal way of interactions: one entity may interact with another one even if the distance between them is not negligible.

As a prototype of the mathematical setting and relationships between three possible scales of description: micro, meso and macro can be kinetic theory of rarefied gases. There is however an important difference: in the case of general (e.g. biological) systems a basic microscopic theory, as the Newton Laws in kinetic theory case, it is not available. Therefore it is reasonable to apply the following strategy. One may start with the deterministic macroscopic model for which the identification of parameters by an experiment is easier. Then one may provide the theoretical framework for modelling at the microscopic scale in such a way that the corresponding models at the macro- and micro-scales are asymptotically equivalent, i.e. the solutions are close each to other in a properly chosen norm. Then, if the parameters of the microscopic model are suitably chosen, one may hope that it covers not only macroscopic behaviour of the system in question, but also some of its microscopic features. The microscopic model by its nature can be richer and can describe a larger variety of phenomena.

We review a general conceptual framework for the program ([1]-[4]) of finding possible transitions between the different levels of description i.e.

(Mi) at the level of'interacting entities (micro-scale),

(Me) at the level of the statistical description of a test-entity (meso-scale),

(Ma) at the level of densities of subpopulations (macro-scale). 
The levels (Mi) and (Me) are of the nonlocal character.

In mathematical terms we are interested in the links between the following mathematical suitable defined structures:

(Mi) the micro-scale of stochastically interacting entities, in terms of jump Markov processes, that lead to continuous (linear) stochastic semigroups;

(Me) the meso-scale of statistical entities, in terms of continuous nonlinear semigroups related to the solutions of nonlinear Boltzmann-type nonlocal kinetic equations;

(Ma) the macro-scale of densities of interacting entities, in terms of dynamical systems related to nonlinear reaction-diffusion equations.

In Ref. [1]-[4] such a conceptual framework was developed for various situations of biological interest. In particular, Ref. [1] deals with the mathematical theory for a large class of reaction-diffusion systems (with small diffusion). Ref. [2] shows that the theory can be generalised to take into account reaction-diffusion-chemotaxis systems (i.e. reaction-diffusion equations with a chemotaxis-type term).

These methods may lead to new and more accurate modelling of complex processes.

\section{References}

[1] M. Lachowicz (2005) General population systems. Macroscopic limit of a class of stochastic semigroups, J. Math. Anal. Appl. 307/2, 585-605

[2] M. Lachowicz (2005) Micro and meso scales of description corresponding to a model of tissue invasion by solid tumours, Math. Models Methods Appl. Sci., 15, 1667-1683

[3] M. Lachowicz (2008) Towards microscopic and nonlocal models of tumour invasion of tissue, Eds. N. Bellomo, M. Chaplain, E. De Angelis, Birkhäuser, Boston 49-63

[4] M. Lachowicz (2008) Links Between Microscopic and Macroscopic Descriptions, in Multiscale Problems in the Life Sciences. From Microscopic to Macroscopic, Eds. V. Capasso, M. Lachowicz, Lecture Notes in Mathematics 1940, Springer, 201-268 